\title{
Redundancy and reducibility in the formats of spatial representations
}

\author{
Sami R. Yousif \\ Version: Accepted, Perspectives on Psychological Science
}

\begin{abstract}
Mental representations are the essence of cognition. Yet, to understand how the mind works, we must understand not just the content of mental representations (i.e., what information is stored), but also the format of those representations (i.e., how that information is stored). But what does it mean for representations to be formatted? How many formats are there? Is it possible that the mind represents some pieces of information in multiple formats at once? To address these questions, I discuss a 'case study' of representational format: the representation of spatial location. I review work (a) across species and across development, (b) across spatial scales, and (c) across levels of analysis (e.g., high-level cognitive format vs. low-level neural format). Along the way, I discuss the possibility that the same information may be organized in multiple formats simultaneously (e.g., that locations may be represented in both Cartesian and polar coordinates). Ultimately, I argue that seemingly 'redundant' formats may support the flexible spatial behavior observed in humans, and that we should approach the study of all mental representations with this possibility in mind.
\end{abstract}

Keywords: spatial representation; format; representation; cognitive map

\section{Introduction}

What is the format of spatial representation? For virtually any spatial property we can think about, there are conflicting answers to this question. Consider the basic idea of a cognitive map, for example. Some theories propose that the format of the cognitive map resembles a Euclidean, Cartesian grid. Other theories propose that the format is non-Euclidean and graph-like. In psychology and cognitive science, the search for the format of spatial representation (or any representation for that matter) is often predicated on the simple assumption that, ultimately, there is a single format to be found in the mind. Must this be the case? One possibility is that the theories of spatial representation that appear to contradict each other do not conflict at all; instead, it is possible that the mind may be formatting the same information in multiple ways simultaneously. Indeed, these seemingly 'redundant' formats may ultimately facilitate flexible spatial behavior. The goal of this paper is to entertain this possibility - to consider the merits of storing multiple 'redundant' formats in principle, and to discuss the evidence consistent with this view in practice. 


\subsection{What is 'format'?}

To represent a piece of information means to store it in a symbolic way, such that it can be accessed, retrieved, and updated (for extended discussions, see Brooks, 1991; Markman \& Dietrich, 2000; Shea, 2018; Thelen \& Smith, 1996). However, representations are not just shapeless bits of information stored in arbitrary units. Representations contain content, and that content must be formatted in some way.

This notion of format should feel familiar, given that it shapes our daily digital interactions. For example, any document could be formatted as a .doc file, or a .pdf file, or a .tex file. Each of these formats has advantages and disadvantages: A .doc file may be better for editing, while a .pdf file may be better for standardized presentation. Ultimately, the entire computer architecture depends on the simple fact that certain programs process specific inputs and produce specific outputs. Those inputs and outputs are representations, and the operations that can be performed on those representations are constrained by their format.

Another example of format is shown in Figure 1. Figure 1A compares the classic game of tic-tac-toe with the "fifteen game". In the fifteen game, there are 9 discs, each representing a digit 1-9. Players take turn selecting one disc from the set. The goal is to end up with a set of exactly three discs that adds up to 15 . The first player to achieve that goal wins. If neither player achieves that goal, it is a draw. (I'll assume readers are familiar with tic-tac-toe.) On their surfaces, these games seem entirely different: one involves a grid with x's and o's, and the other involves summing numbers to fifteen. Yet when those numbers are superimposed on the tic-tac-toe grid in a certain order (see Figure 1A), you can see their similarity. Just like tic-tac-toe, the "fifteen game" contains a finite number of solutions. Just like tic-tac-toe, there are exactly nine possible moves, and some are better than others: picking both "5" and the central square in the grid result in the maximum number of winning combinations (where the numbers "3", "1", "7", and "2", like the left, right, top, and bottom squares result in the fewer number of winning combinations). And, just like tic-tac-toe, participants take turns making selections, and a 'winning' combination requires exactly three selections. The difference between these two games could be understood as a difference in format: tic-tac-toe traffics in x's and o's, whereas the fifteen game traffics in numbered discs. Functionally, the inputs and outputs of the two games are equivalent, yet their different formats constrain how we interact with the information.

The history of cognitive science has been shaped by questions of format. Many of the field's most prominent debates have centered around this exact issue. For example: is imagery depictive (see Kosslyn et al., 1995; Kosslyn, 1996) or propositional (see Pylyshyn, 1973; Pylyshyn, 2002)? Are there a finite number of dimensions of 'face space' underlying face perception (e.g., Chang \& Tsao, 2017)? What latent structures form the basis of human language (e.g., Traxler \& Gernsbacher, 2011)? In each of these cases, understanding the format underlying these representations reveals something about how the mind solves 


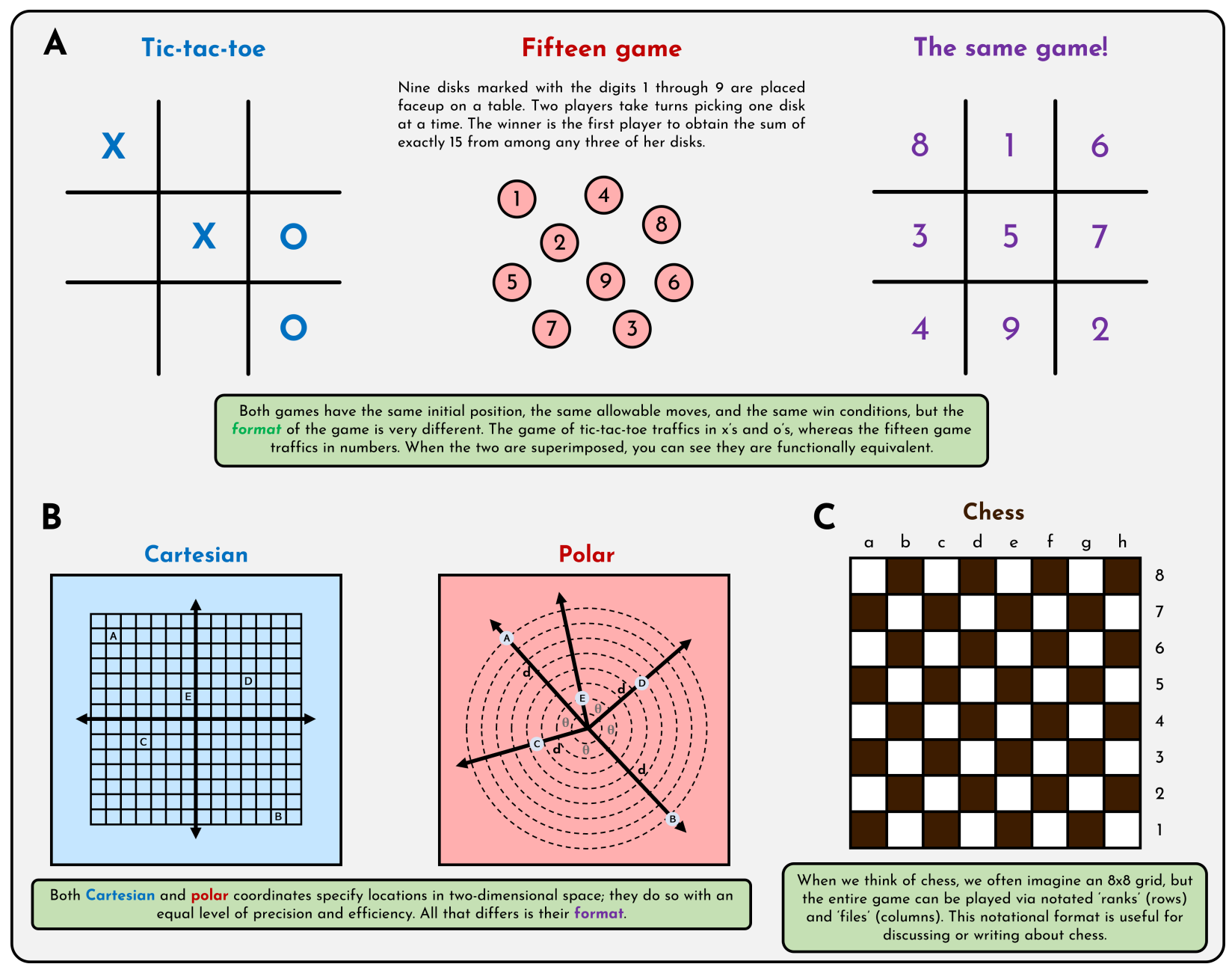

Figure 1: Examples of format. (A) A comparison between the classic game of tic-tac-toe and the lesser known 'fifteen game'. One may say that these games differ in their 'format'. (B) A comparison of a classic distinction between canonical spatial formats: Cartesian coordinates and polar coordinates. (C) Even the game of chess can be played in multiple 'formats'!

these critical problems (of imagery, face perception, and language) in the first place.

There is much to say about what 'format' means precisely. The goal of this paper is not to make any strong claims along these lines; it is to discuss 'format' in the broadest possible sense, sidestepping these nitty-gritty semantics for now. However, it may be helpful to keep in mind Marr's three levels of analysis as a reference point (Marr, 1982; see also Maley, 2021 for a useful and thoughtful discussion). The highest level, the computational level, describes what is being represented. The lowest level, the implementation level, describes the physical substrate on which that computation is implemented (e.g., in animals, neurons). And at every step in between the computational level and the implementation level — all the intermediate levels that may be collectively referred to as the representational/algorithmic level — information must be organized in some way. That organization, whatever it may be, is 'format'. 


\subsection{One format or many?}

Although we often think of representations as being formatted in only one way, the goal of this paper is to consider the possibility that some representations may be formatted in multiple different ways simultaneously. Why would storing information in multiple formats be useful in the first place? First, think of the number 'seven'. In base-10, the quantity 'seven' can be represented by the digit 7 . However, in base-2, the quantity 'seven' is represented by the digits 111 . In base-3, by the digits 21 . We can think of these different bases as formats. It is not as though one base or another captures the quantity 'seven' any more precisely; they just represent the quantity in different ways. Each format may have its own merits: formatting information in base-10 is intuitive to most people, but formatting information in base-2 is a necessary feature of modern computers.

Second, think about the game of chess. To most people, a chess board is nothing but an 8 by 8 grid. If you want to make a move - or explain a move - you must refer to that grid. Indeed, this is the most natural way to see and play the game of chess. But it is not the most natural way to talk about the game of chess. Experienced chess players and commentators use specific notation (in modern chess, 'algebraic notation') to talk about the movement of pieces (see Figure 1C). For example, if you have the white pieces and you want to move the pawn in front of your king two spaces forward, you could explain that in words, or you could simply say "e4". Using this notation requires not even one full word to convey the same move that was just described using 23 words. The algebraic notation is useful: it simplifies communication about the game of chess. That said, it would be hard to learn the game of chess from the algebraic format alone. The (beautiful) geometry of the game is most apparent when viewed as an 8 by 8 grid.

This is to say that each format serves a unique function; mastering chess requires understanding both formats. However, neither format is strictly necessary. The 'Stockfish' chess engine, for example, need not understand the game of chess as a grid at all. It must only represent the statistical value of a given move to a given tile, regardless of where that tile is relative to the other tiles. Conversely, most casual players of the game never interact with the algebraic notation. For veteran players of the game of chess, though, there is value in being able to translate seamlessly between formats as needed. This is why 'redundant' formats may be useful: Human minds are not machines designed to compute specific tasks optimally. The reason we have algebraic notation in the first place is because it enables us to explain and describe the game (succinctly) in written form. Yet, it is not as if the development of chess notation forced chess players to abandon the board altogether; we still enjoy and appreciate the game in its physical, two-dimensional form. Unlike chess engines, it benefits the human mind to represent the chess board in multiple ways. Whereas simpler organisms (and chess engines) may benefit from having one highly specialized system for each of its 
(few) behaviors, more complex organisms may be better served by cognitive systems adapted to complete innumerable tasks flexibly. As such, it may benefit certain minds (in this case, chess experts) to represent information redundantly - in multiple formats, which may be called upon separately depending on the task at hand. There is precedent for this notion of 'redundant' formats in cognitive science. Classically, dual coding theory posits that any given piece of information can be formatted both visually and verbally (for review, see Paivio, 1991). The concept 'chess', for instance, may have a visual form (e.g., an image of a chess board and its pieces) and a verbal form (e.g., explicit knowledge about the organization of the chess board and where each piece belongs). Here, the adaptive value of redundancy is obvious: If the mind has twice as much information to remember, it may reduce the likelihood of forgetting. Further, if any one kind of information is lost (whether due to forgetting, or some serious brain trauma), the other may be preserved. Thus, 'redundant' formats may not only serve different functional purposes; they may also serve as a much needed 'backup' in cases of emergency.

\subsection{The format frontier: Space}

This paper focuses on the formats of spatial representation as a 'case study', for a few reasons. First, space is perhaps the single domain of cognition for which the most is known about the format of mental representations, and so serves as an apt case study of mental representation more broadly. Second, spatial representations may themselves serve as the format for representing other higher-level information, whether that be numerical information or social information (for review, see Peer et al., 2020). As such, understanding the formats of spatial representations may in turn reveal the format of other representations. Finally, spatial representation is ubiquitous in the animal kingdom: virtually all organisms depend on representations of space in one way or another (where far fewer may possess imagery, or sophisticated face perception, or language).

The purpose of this article is to discuss the different 'formats' of location representations (a) across species and across development, (b) across spatial scales, and (c) across levels of analysis (e.g., high-level cognitive format vs. low-level neural format), while briefly commenting on the format of other spatial properties (e.g., shape and size). Along the way, I will highlight many instances where there are multiple viable candidate formats of a given representation. I argue that while we often think of spatial representations as being reduced to a single format, it may be fruitful to consider whether the mind represents spatial information in multiple formats simultaneously. Although format-redundancy may be inefficient in some respects, it may also contribute to the flexibility of spatial behavior observed in humans and in some non-human animals. 

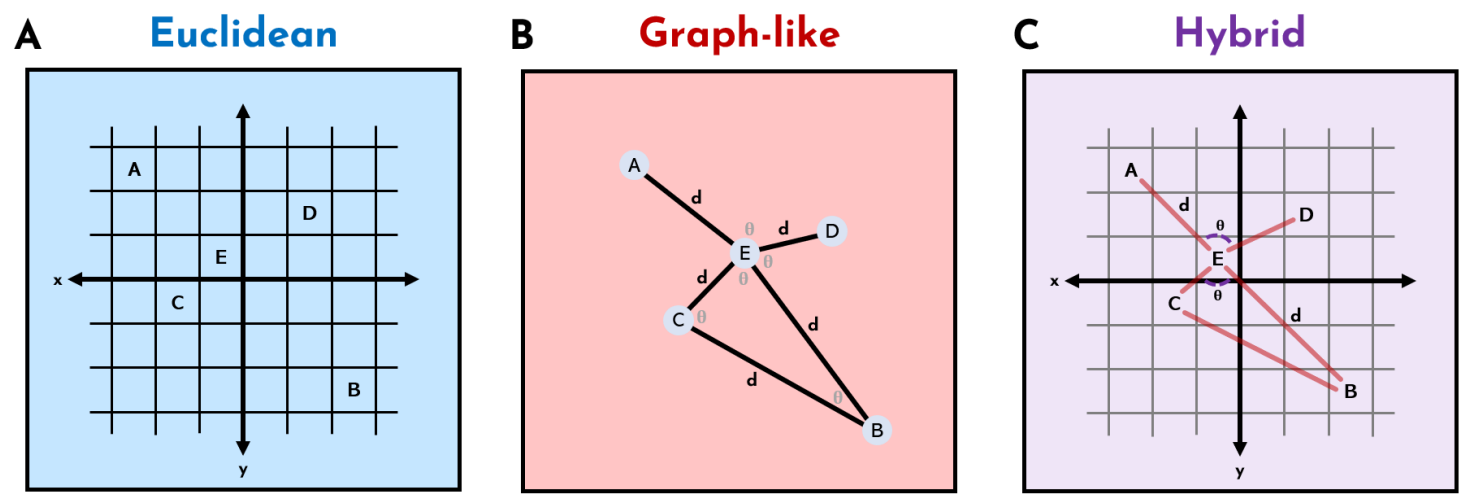

Figure 2: Examples of models of large-scale representation.

\section{The Representation of Location}

The most fundamental piece of spatial information we represent is location; indeed, most other information we could represent would be quite useless if we were not first able to represent where things are. Perhaps for this reason, an explosion of research at the beginning of the cognitive revolution took an interest in the representation of location. The earliest incarnation of these ideas was the 'cognitive map' (Tolman, 1948). As the name suggests, this theory held that the mind internally represents the spatial structure in the world, analogous to a physical map. But what is the format of this cognitive map?

\subsection{Large-scale spatial representation}

Early theories proposed that cognitive maps are roughly Euclidean (e.g., Gallistel, 1990; O'Keefe and Nadel, 1978). These models described a metric, two-dimensional vector space over which other information could be placed. On this view, if you wanted to travel from your office to your favorite coffee shop, you would call to mind your cognitive map, locate the coffee shop upon it, and extract the spatial coordinates attached to it. Then, separately, you would perform some computation to determine the optimal route from one location to another. The advantage of this format is that a large-scale environment can be represented in a single two-dimensional vector space; as such, new information can be easily integrated into a map as you explore. For example, if you discover a new coffee shop just outside of town, you'd just need to add that shop's location on your now-expanded map of the area (see Figure 2A).

A popular alternative view is that locations are represented in a network-like or graph-like fashion; for example, one may imagine a web of connections between points of interest (see Figure 2B; Kuipers, 1978; Kuipers, 1982; Warren et al., 2017). On this view, if you wanted to travel from your office to your favorite coffee shop, you would not need to call to mind your cognitive map of the surrounding area. Instead, you 
would need only call to mind two nodes in that network (your office and the coffee shop) and the edge between them. Network models like these are often explicitly non-Euclidean. Formally, this means that knowing the relation between $\mathrm{A}$ and $\mathrm{B}$, as well as the relation between $\mathrm{B}$ and $\mathrm{C}$, does not allow one to compute the relation between A and C. In practice, this means that points are being represented in a non-uniform space.

Some work provides direct support for the network-like view of spatial representation. Warren and colleagues (2017) had participants complete an unusual task: they had to navigate within virtual, nonEuclidean spatial environments. In other words, participants navigated through mazes with 'wormholes' interspersed; these wormholes teleported participants from one point in the space to another (see Figure 3B). Following training in the environment, participants were taken to a landmark and asked to point in the direction of another. The critical measure was the distortion of participants' pointing errors. As can be seen in Figure 3A, participants who navigated the Euclidean space pointed accurately at the target; however, as can be seen in Figure 3B, participants who navigated in the non-Euclidean space pointed in a systematically distorted way - as if they failed to represent the entire space on a Euclidean map and instead represented the space based on their experience navigating. Thus, Warren and colleagues argue that these pointing errors revealed evidence of a network-like representation instead.

This network view is supported in part by a body of work indicating that people's large-scale spatial representations are viewpoint-dependent (e.g., Shelton \& McNamara, 1997; Waller et al., 2002). That is, environments are better remembered from the perspective they were learned, suggesting that individuals fail to form a truly robust, abstract spatial map that is invariant across perspectives.

Some have argued that spatial representation cannot be explain solely by either Euclidean, Cartesian models or graph-like models. For example, Chown et al. (1995) argue that wayfinding should be understood not as a highly specific, modular process, but as one that is integrated with higher-level cognition; as such, their model incorporates numerous aspects of spatial information (rather than merely a single coordinate system). Along similar lines, Tversky (1993) argues that spatial representation is best understood not as the product of a cognitive map, but as a 'cognitive collage', integrating many different information types into complex representations of space.

It may well be the case that the mind integrates many kinds of information to form a 'cognitive collage' of space. Still a question arises about how location information within those collages is formatted - and whether any sort of coordinate representation plays a role. For example, there are models that combine features of Cartesian and network-like models (e.g., Meilinger, 2008; Poucet, 1993; see Figure 2C for a simplified example); these models are more specific than the 'cognitive collage' theory in that they still posit reliance on some coordinate representation. Other theories are even more explicit. For example, Peer et al. (2020) raise the possibility that Euclidean cognitive maps and network-like cognitive graphs can be learned 
A
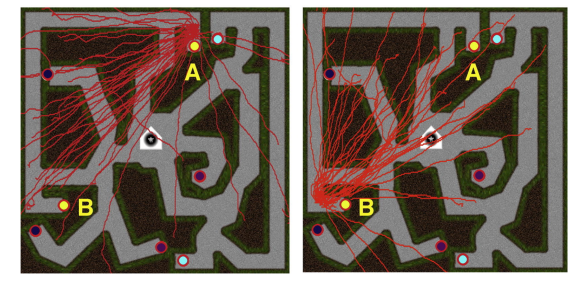

B
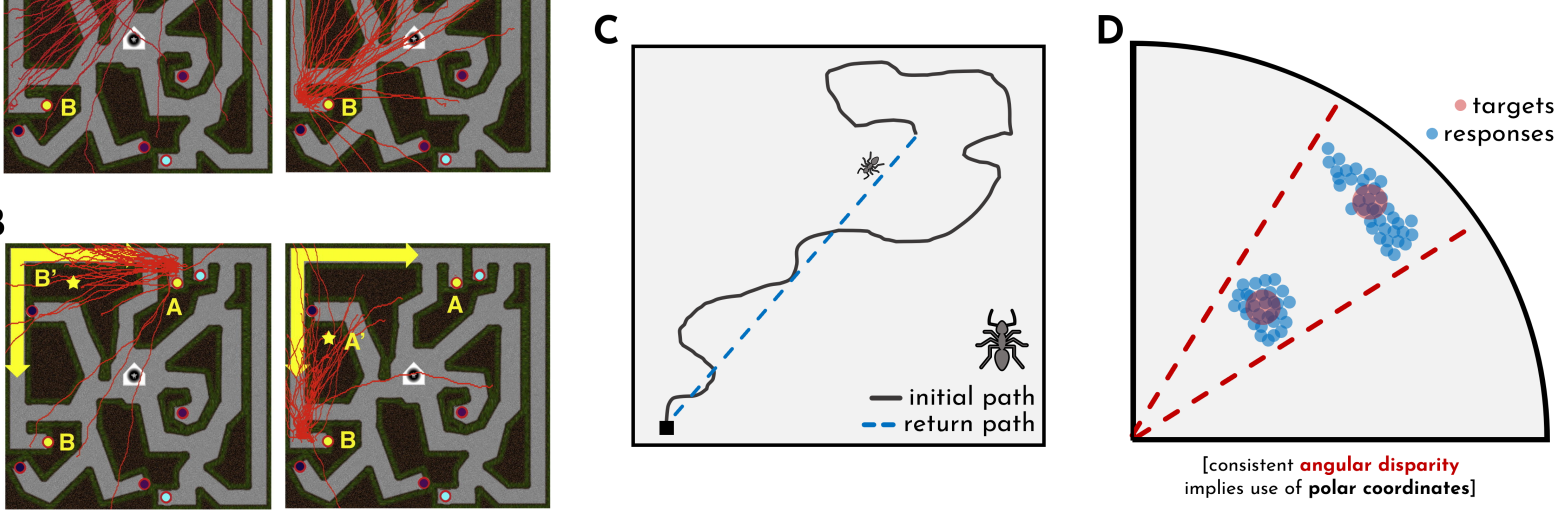

G

E

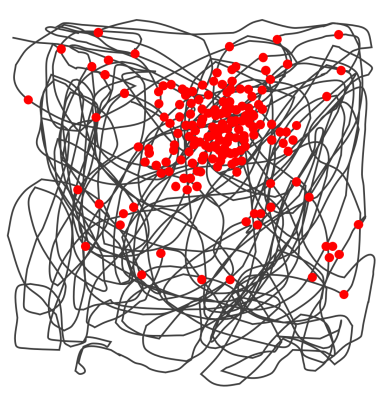

$\mathbf{F}$

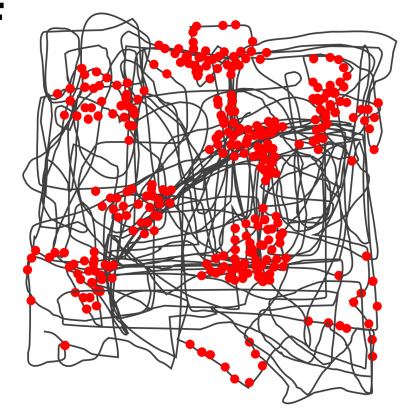

Figure 3: Pointing errors following navigation in (A) a Euclidean space or (B) a non-Euclidean space (in which the yellow line represents a 'wormhole'), adapted from Warren et al., 2017. (C) Caricatured depiction of navigation in cataglyphis fortis. (D) Evidence for polar coordinates in localization errors, adapted from Huttenlocher et al., 1991. Caricatured depictions of (E) place cells and (F) grid cells, modeled after Moser et al., 2008. (G) A depiction of cognitive map distortions: nearer areas are allocated increased 'representational space' relative to farther areas.

separately, but simultaneously, each perhaps serving different spatial goals.

To date, though, behavioral evidence that multiple formats are represented simultaneously is scant (but see Yousif \& Keil, 2021b) - in part because much empirical work has implicitly committed to the idea that location is represented in only one format. This view may limit empirical progress, however. There is a more foundational question to answer: Are these formats mutually exclusive in the first place? If not, experiments designed to tease apart different formats may be misguided. 


\subsubsection{A 'case study' from animal behavior: Desert ants}

Studying navigation behavior in humans is complex. For example, how can we tease apart implicit systems of spatial representation from explicit, linguistic strategies? For this reason, simpler organisms, like rodents and insects, often offer more direct insight into the nature and format of spatial representations. Work on certain species of desert ants (cataglyphis fortis), for instance, has revealed highly specific navigation behavior: These ants always return home along straight paths, even if they foraged along a circuitous route (Müller \& Wehner, 1988). How?

It has been suggested that these ants have a built-in 'pedometer' that tracks their steps. This, along with a sense of direction, is sufficient for them to find their way home, even after traversing long distances (Wittlinger, Wehner, \& Wolf, 2006; see Figure 3C). To demonstrate that ants rely on step counts, Wittlinger and colleagues (2006) placed stilts of different lengths on ants, such that each step covered a greater distance. In another manipulation, they shortened the legs of some ants. Their hypothesis was simple: if ants navigate using some sort of step counter, they should overshoot the nest when their legs are longer and undershoot the nest when their legs are shorter. This is exactly what they found.

This example speaks directly to the issue of format. Of all the pieces of information these ants may have represented, they appear to have represented only two: direction and steps. Thus, we might say that the 'format' of the desert ant's location representations is a two-dimensional vector containing a variable for direction and a variable for step number. Fortunately for the ants, this is all the information one needs to create a vector between two points in space; thus, with very little information, the ants are able to navigate directly back to their homes. This is the holy grail - near-direct insight into the underlying format of a representation via a simple, elegant behavioral intervention.

That said, while this stands out as a powerful example of format in an animal mind, it also stands out as a powerful example of how any single format may be insufficient to support flexible, complex behavior. Desert ants, unlike humans, exhibit a narrow range of spatial behavior: They navigate only in one kind of environment and rely upon stable sources of information in that environment (like the sun). And desert ants, unlike humans, are easily confused: If you simply put the ants on stilts, they will happily navigate right past their destination. Humans, in contrast, can successfully navigate virtually any terrain; they can do so in light, or in darkness; and they can almost effortlessly translate information from one scale (say, a map) to another (say, the environment that map represents). This more complex behavior may require a capacity for representing (and integrating) information in multiple formats simultaneously. 


\subsubsection{Development}

The format of spatial representation may occasionally reveal itself through developmental change. For example, children may have some systems (vector-based, or coordinate-based, like desert ants) that are more rapidly developing, and others (e.g., 'cognitive collages') that are later developing. Understanding how multiple sources of information are integrated into spatial representations may require a developmental lens.

Inspired by classic work with rats (Cheng, 1986), a substantial body of work has investigated children's sensitivity to the Euclidean geometric features of an environment via 'reorientation' tasks (see, e.g., Hermer \& Spelke, 1994; Lee, Sovrano \& Spelke, 2012; Yousif \& Lourenco, 2017; for reviews, see, Cheng \& Newcombe, 2005; Twyman \& Newcombe, 2010). Some evidence suggests that, after disorientation, children will use some geometric cues (e.g., distance, direction) but not others (e.g., angle, length) to reorient themselves (Lee, Sovrano, \& Spelke, 2012; although more recent evidence suggests that children may have access to a wider range of cues, Yousif \& Lourenco, 2017). Such work is concerned with sensitivity to geometric features of the external environment rather than the internal coordinates used to represent locations. However, the fact that children use distance and direction information to reorient may speak to format: we might say, for instance, that children's cognitive maps are formatted with distance and direction information, but not angle or length. Furthermore, there is evidence that young children will successfully localize objects in space, even before they learn to superimpose categorical structure on environments (Huttenlocher et al., 1994). This may suggest that coordinate-based representations are earlier developing, and that more explicit spatial strategies develop more slowly throughout childhood.

There is considerable debate about whether reorientation tasks reflect large-scale map representations. Children's reorientation behavior varies depending on the physical size of the space (see, e.g., Learmonth et al., 2008), although they generally tend to rely on similar geometric cues across scales. This debate raises questions about what kinds of environments trigger the use of 'cognitive maps' in the first place - and, again, hints at this notion that the entirety of spatial behavior cannot be boiled down into a single format.

\subsection{Small-scale spatial representation}

So far, spatial representation has been discussed on the scale of navigable environments. However, such spatial representations will not help us to reach for our coffee, or catch a baseball, or drive along a busy highway. For that, we must represent spatial information on a smaller scale. What are the formats of location representations of visual and motor coordinates, for example? 


\subsubsection{Visual coordinates}

The format of visuospatial representation has been a topic of interest for decades. There is a long tradition, beginning with Huttenlocher and colleagues (1991), of using errors in spatial representation to evaluate format (see also Wittlinger et al., 2006). Huttenlocher and colleagues had observers complete a simple spatial memory task in which they saw a dot present somewhere inside of a circle and had to recreate the location of that dot. They found systematic errors: observers frequently (mis-)placed points closer to the center of the quadrant in which it originated (or, as they called it, the 'prototype'). Based on this evidence, the authors made several important claims about representational format. First, they distinguished between coarse and fine-grained representations. Whereas the former, they argued, may be categorical (i.e., in the top-left vs. the bottom-right), the latter may depend on a sort of coordinate system. Second, they made a claim about what the coordinate system supporting fine-grained spatial representation might be; they tentatively suggested that locations may be represented in polar coordinates (see Figure 3D). In other words, they argued that the 'format' of spatial representation is more than a single two-dimensional vector; instead, they argued that spatial information may be 'formatted' in different ways at different spatial scales. This notion of coarse, categorical spatial representations (often called the 'Category Adjustment Model', or CAM) is still popular today; in one instance, for example, it has been shown that the CAM explains errors in three-dimensional spatial judgments as well (suggesting this model has value as a 'general' model of spatial representation across both two-dimensional and three-dimensional forms; Holden et al., 2013).

More recent work has homed in on the question of visual coordinates specifically. Using a similar dotplacement task, Yousif and Keil (2021b) analyzed the errors observers made when localizing objects. The aim of this approach was to infer format from systematicity in observers' errors.

How could localization errors reveal format? Imagine that the mind represents localization via some two-dimensional vector (it need not, but suppose that it does). Those two dimensions should be orthogonal; otherwise, the representation would be inefficient. As such, the errors in those two dimensions should also be orthogonal; if the mind represents two dimensions independently, there is no reason that an error in one dimension ought to influence the error in another (indeed, this would lead to dramatically suboptimal behavior).

To make this more intuitive, consider a silly example. Imagine that you represent all people on a twodimensional vector of [tallness, niceness]. Naturally, your memory is fallible, so you will not remember exactly how tall or exactly how nice every person is. But if these are two unrelated dimensions, then you should not mistake tallness for niceness. In other words, if you misremember someone as being taller than they really are, that should not influence how you remember their niceness. If it did, that may suggest 
A. Method: Localization task

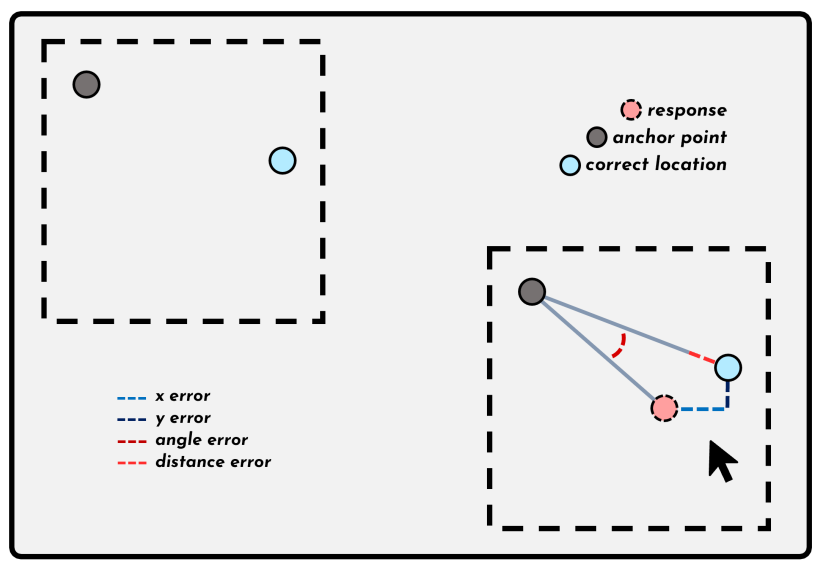

\section{B. Logic}

An efficient two-dimensional format should be compromised of two independent, orthogonal dimensions. As such, errors in that format should also be independent from one another. So, here, if we find a positive correlation, it indicates that errors are not independent and therefore that the coordinate system is an unlikely candidate for format.

\section{C. 'Error correlation' analysis}

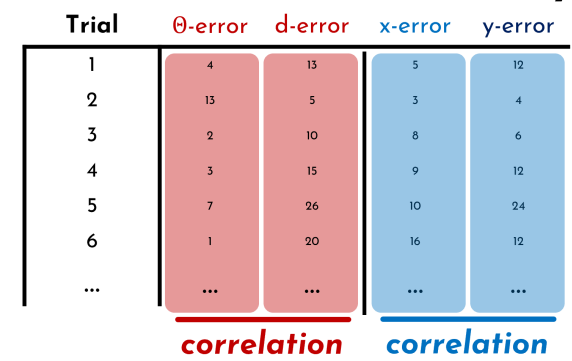

(depiction of the basic design/analysis from Yousif \& Keil, 2021)

Figure 4: Visual explanation of the 'error correlation' analyses, adapted from Yousif and Keil (2021b).

you are not representing 'tallness' and 'niceness' independently. The same logic applies here: if angle and distance are two separate dimensions of a location representation, then they should be uncorrelated. Across several paradigms, that is what Yousif and Keil (2021b) find: Cartesian errors (i.e., the errors made in the $\mathrm{x}$ and $\mathrm{y}$ dimensions) were consistently correlated, whereas polar errors (i.e., the errors made in the angle and distance dimensions) were consistently uncorrelated (see Figure 4). This pattern suggests that observers relied on polar coordinates to complete these tasks.

However, human errors can, in some contexts, reveal Cartesian-esque behavior. Most notably, when observers perform a localization task over a visible grid, their errors shift to favor Cartesian coordinates (i.e., errors between the $\mathrm{x}$ and $\mathrm{y}$ dimensions become uncorrelated; Yousif \& Keil, 2021b). The findings hint at the possibility that spatial representations operate flexibly in multiple formats simultaneously. In unstructured environments, perhaps polar (or graph-like) formats are used by default. Conversely, in structured environments (e.g., perhaps a grid-like city structure), perhaps Cartesian-esque formats are more useful. The mind could support both types of representations simultaneously, calling upon one or the other depending on the needs of the task.

Although Huttenlocher and colleagues (1991) and Yousif and Keil (2021b) both used visual tasks, the work highlighted so far does not speak to visual coordinates per se. (Indeed, both papers make points about spatial representation in general, rather than about visuospatial representation itself.) It is necessary to disentangle visual coordinates from external spatial coordinates. There is some evidence that eye movements themselves may operate in polar coordinates. For instance, Robinson (1972) demonstrated that the distance and direction of saccades (in monkeys) following stimulation of the superior colliculi were uncorrelated 
(implying independence). Interestingly, this suggests that the format of high-level cognitive representations of space may originate, in part, due to low-level physiological constraints (insofar as they may be 'wired in' to the physical movements of eyes). Indeed, some recent work in humans has also suggested that vision itself may rely on polar coordinates (e.g., Yang \& Flombaum, 2018).

\subsubsection{Motor coordinates}

Actions like reaching for an object require integration of two kinds of spatial information. First, one must perceive the environment and represent that information in some way. Second, one must initiate a motor action that itself requires a representation of space. How does the mind represent location for purposes of action?

Interestingly, there is evidence that motor actions, like visual space, may also depend on polar coordinates. As early as 1992, Flanders and colleagues argued for a polar coordinate system originating at the shoulder; this conclusion has been supported by subsequent work (Baud-Bovy \& Viviani, 1998). Other work on planar movements measured the distribution of errors for reaches to target locations and consistently found elliptical patterns of errors along axes stemming from the origin (similar to Huttenlocher et al., 1991; Gordon et al., 1994). Such a pattern implies that angle and distance are represented independently (insofar as each dimension reveals distinct patterns of errors), and therefore that polar coordinates are a likely candidate coordinate system for motor actions.

Some work suggests that the nervous system encodes location information in both a Cartesian and polar format (depending on the demands of the task at hand; Hudson \& Landy, 2016). This latter idea is consistent with the notion that large-scale spatial representations may too be represented in both Euclidean and graph-like formats (Peer et al., 2020), and that spatial information may be represented in multiple formats simultaneously (see also Chown et al., 1995; Meilinger, 2008; Poucet, 1993).

The motor system may rely on coordinate representations - but are these the same coordinate representations used by the visual system? Some work has suggested that there may indeed be a shared representational format. Specifically, it has been argued that posterior parietal cortex may be responsible for the translation between visual and motor coordinates (Andersen et al., 1985; Andersen \& Zipser, 1988; Xing \& Andersen, 2000). However, these proposals make no specific claim about what coordinate systems are used to support visual and/or motor coordinates. 


\subsection{Neural representation of location}

Ultimately, representations must be realized on some physical substrate (i.e., at the implementation level; in this case, the brain). How do the physical structures in the mind relate to format?

\subsubsection{Place cells, grid cells and head-direction cells}

Three primary structures have been identified that may support coordinate representations in the brain. The first, described in 1971, are 'place cells' (O'Keefe \& Dostrovsky, 1971; see Figure 3E). These cells selectively fire in response to specific 'places' within a space (hence their name), suggesting that they may serve as the most basic units of a 'cognitive map'-like representation (see O'Keefe \& Nadel, 1978). The second type is 'head direction cells'. As the name implies, these cells are thought to represent an organism's heading direction in space (for review, see Taube, 1998). Head direction cells, in combination with place cells, were thought to be sufficient to support complex spatial behavior. However, decades later, a third type of cells was described: 'grid cells' (Hafting et al., 2005; see Figure 3F). Unlike place cells, grid cells fire not just in response to a single location, but periodically throughout a space in a stereotyped triangular/hexagonal structure. Some evidence suggests that grid cells are the basic format of visuospatial representations (Julian et al., 2018), and may even support object, face, and scene perception (Bicanski \& Burgess, 2019). Combined, these cells are thought to form the basis of all spatial representation and spatial behavior (see Moser et al., 2008).

A question arises, then, about how these neural representations support or are related to the cognitive models of spatial representation. One view is that grid cells in particular seem to support Euclidean spatial representation (consistent with some proposals of the organization of the 'cognitive map'; e.g., Gallistel, 1990; O'Keefe \& Nadel, 1978). However, there is no concrete evidence linking the two (but see, e.g., Chen et al., 2015). It is not impossible to imagine how the hexagonal structure of grid cells - depending on their exact resolution - could support network-like (Kuipers, 1978, 1982; Warren et al., 2017) or polar (Huttenlocher et al., 1991; Yousif \& Keil, 2021b) representations, especially in combination with head direction cells (Taube, 1998). To understand spatial behavior, then, a key goal for cognitive neuroscientists moving forward would be to better understand the links between the low-level neural format and the high-level cognitive format of our most primitive spatial representations.

\subsubsection{A 'case study' from animal behavior: Non-Euclidean cognitive maps in rats}

It has long been known that people's cognitive maps seem to be distorted: Familiar areas are overrepresented relative to unfamiliar areas, for instance - resulting in a sort of dilation near the focal regions of a given 
cognitive map (i.e., the area within one mile of your home may occupy 10 units of representational space, whereas the area within one mile beyond that may occupy only a few units of representational space; Figure 3G; see Holyoak \& Mah, 1982; Sadalla et al., 1980). This fact is at odds with the notion that grid cells support a Euclidean cognitive map. If grid cells fire periodically, how could a cognitive map become distorted?

Recent work in rats has shown that grid cells themselves can be 'remapped' flexibly depending on the context; specifically, when rats navigate in environments with rewards, areas near rewards become overrepresented relative to regions farther from rewards (resembling distortions observed in humans; Buter et al., 2019). In environments with no rewards, grid cells reveal a more regular pattern of firing. This suggests that even these cells - in many respects, the most promising sign of a Euclidean cognitive map - may support distorted, non-Euclidean representations of space.

\subsection{Propositional representations of space}

Are coordinates the only viable formats of spatial location? Imagine giving directions to a friend. You might say, "The coffee shop is down that road, after the market but before the post office." You'd be able to locate the coffee shop without representing its absolute location in space. And with enough landmarks, you'd be able to represent locations with fair precision. Imagine that the coffee shop is between the market and the post office in the North-South dimension, and between the theater and an office building in the East-West dimension; you could represent all of these locations as part of a propositional network, without needing to represent the precise angles/distances between them. Indeed, some work has posited that spatial representation is possible via propositional representation (Pylyshyn, 1973). This idea that space is represented propositionally is consistent with some proposals that the mind represents location in a coarse (e.g., "that point was generally up and to the left"; Huttenlocher et al., 1991), hierarchical (Huttenlocher et al., 1994; McNamara et al., 1989; Taylor \& Tversky, 1992), or relational (Jiang, Olson, \& Chun, 2000) manner. There is also work demonstrating strong angular biases in simple navigation tasks, such that individuals have a strong tendency to misremember angles as closer to 90 degrees than they were (Sadalla \& Montello, 1989). Such a tendency may indicate a bias to 'orthogonalize' paths, perhaps as part of explicit, propositional representations of space.

That said, it is likely that coordinate systems underlie spatial representation at least to some extent, at some scales. For example, certain individuals with localization deficits exhibit patterns of errors that may best be explained by appeal to a coordinate system. Most famously, patient AH frequently "mirror flipped' points in space (McCloskey et al., 1995; McCloskey \& Palmer, 1996). That is, AH may misplace to the left a point that they had seen on the right; the relative location would be the same, though (as if 
the 'sign' of one dimension had 'flipped', but the representation was otherwise preserved). Thus, an open question remains about the pervasiveness of coordinates as a means of representation. On the one hand, it is possible that the fundamental unit of spatial representation is some kind of coordinate system, but that higher level relational reasoning leads to configural/relational processing (as in Jiang et al., 2000). On the other hand, it is possible that coarse representations (see Huttenlocher et al., 1991) and fine-grained coordinate representations (see Yousif \& Keil, 2021b) operate in tandem. There is evidence that supports this possibility. Kosslyn and colleagues $(1989,1992)$ argued that the mind separately represents both categorical and coordinate relations (and that the left hemisphere is specialized for processing the former, whereas the right hemisphere is specialized for processing the latter). This is yet another body of evidence that hints at the notion that representations of location may not be reducible to a single underlying format.

\subsection{Spatial location as format}

The examples so far fail to capture the full extent to which we depend on spatial representations. For example, our representations of number are also thought to be fundamentally spatial (e.g., Aulet et al., 2021; Dehaene et al., 1993). Social relations may be represented spatially (Parkinson \& Wheatley, 2013). For that matter, virtually all information represented in working memory may be retained in a spatial way (e.g., van Dijck et al., 2014; van Dijck \& Fias, 2011; Yousif, Rosenberg, \& Keil, 2021). Thus, we might say that the format of numerical representations (or social representations, or representations maintained in working memory) is (are) spatial (at least to some extent). And in each of these instances, a question arises about the format of the underlying spatial representations. Do we represent numbers in a Cartesian space? Do we represent social relationships in a polar-esque cognitive graph? Given that non-spatial knowledge may be represented in a spatial way, understanding the format - or formats - of spatial representation may help us to understand not only spatial representation, but all representation.

This point was recently articulated by Peer and colleagues (2020) who described how non-spatial information could be represented using either Euclidean or graph-like cognitive maps. They write, "...we may represent the people we know in terms of continuous variables such as various abilities that are naturally encoded as a map-like attribute space... or we may represent them in terms of discrete relationships between individuals that are naturally encoded in graph-like formats (e.g., social networks or family trees)" (p. 48). Indeed, some work has shown that hippocampal cells responsible for spatial representation in rodents do encode other dimensions like time (e.g., MacDonald et al., 2011). Perhaps more interestingly, fMRI has revealed Cartesian-like structures for the representation of numerous stimulus dimensions, including some concrete features like visual size and opacity (Theves et al., 2019), as well as more abstract features like 
popularity and competence (Park et al., 2020). Nevertheless, there is no clear example of any abstract form of knowledge being represented in a graph-like or polar-esque way. (This does not mean that Cartesian coordinates better format for abstract information, or even the more common format. Perhaps, given their computational simplicity, such representations are just easier to detect.)

Understanding spatial representation continues to be a promising way forward for understanding the format of mental representations in general.

\section{Abstract, Generalized Spatial Representations}

When thinking about the format of spatial representation, it is of the utmost importance that we think about what information is preserved across scales / modalities. Imagine how challenging it would be to read a map, for example, if the mind struggled to translate between visuospatial representations and environmentalspatial representations. Or how challenging it would be to reach for a light switch in the dark. Human spatial behavior is incredibly flexible, and flexible behavior requires flexible, generalizable spatial representations. This raises a question: Is there any evidence of a generalized, stable format of spatial representations across scales and modalities?

The short answer is "no". For example, some work has argued for a distinction between small-scale and large-scale spatial behavior, insofar as individuals display no stable spatial abilities that transcends task type (Hegarty et al., 2006). However, just because different levels of spatial representation are dissociable does not bear on whether they rely on a common format. Indeed, there is mounting evidence across a range of approaches that one of two formats - or, both - may underlie spatial representation across scales. The first possible format is a sort of Euclidean, Cartesian map (see, e.g., Gallistel, 1990; O'Keefe and Nadel, 1978). This format would consist of a two-dimensional vector that roughly captures horizontal and vertical extent. The strongest evidence in support of this possibility comes from the fact that grid cells, one of the fundamental units of spatial representation, could support Euclidean format (Hafting et al., 2005). This model is valued for its flexibility, i.e., the fact that it allows for new information to be incorporated easily as a 'cognitive map' expands. However, the evidence in support of Cartesian maps is primarily restricted to large-scale, environmental coordinates; there is little evidence, if any, that Cartesian maps are used to represent visual space, for example (but see Peer et al., 2020, for discussion of how other forms of information may be formatted in a Cartesian-esque way; and see Yousif \& Keil, 2021b for one possible exception).

The second possible format is a polar, or graph-like, format. This format would consist of a twodimensional vector that roughly captures angle and distance. Evidence of polar coordinates has been found in vision (Robinson, 1972; Yang \& Flombaum, 2018), in motor movements (Baud-Bovy \& Viviani, 1998; 
Flanders et al., 1992; Gordon et al., 1994), in small-scale, non-navigable environments (Huttenlocher et al., 1991; Yousif \& Keil, 2021b), and in large scale, navigable environments (Kuipers 1978, 1982; Warren et al., 2017). Thus, polar coordinates would seem to make an excellent candidate for a flexible, abstract, general form of spatial representation.

Although this does not mean that the mind must represent only one format across scales (indeed, I have argued the opposite - that the mind may be representing multiple kinds of information at once), it would be concretely useful for the mind to represent at least some information in a common format across scales. Otherwise, every ordinary task would require tedious translation back and forth across formats. Thus, the abundance of evidence supporting the use of polar coordinates (and/or graph-like models of space), is notable: Even if the mind represents other forms of information, it may be that polar coordinates are especially well-suited for translating across scales and modalities.

\subsection{One Format, or Many?}

There are many distinct cases where the available evidence suggests that spatial information may be simultaneously represented in multiple formats. For example, there is evidence that large-scale (environment-level) spatial representations are supported by both Cartesian-esque (e.g., Gallistel, 1990; O'Keefe and Nadel, 1978) and graph-like (e.g., Kuipers, 1978; Kuipers, 1982; Warren et al., 2017) formats; the available neural evidence is consistent with both of these possibilities. For small-scale, visuospatial representations, too, there is evidence that people may flexibly represent space in multiple formats, depending on the constraints of the task (Yousif \& Keil, 2021b).

This is not to say that the mind merely represents different kinds of information at the same time. It is virtually undeniable — and perhaps trivially true — that different kinds of spatial information can be represented simultaneously. For example, you may have a sense of the space of your surrounding area in terms of the spatial layout itself and in terms of landmarks/street names/etc. These are different kinds of information that may or may not be integrated into a single representation (but see Tversky, 1993).

But there is a more radical possibility: that we may represent the same kind of information, at the same level, in multiple formats simultaneously. Note, for instance, that Cartesian coordinates and polar coordinates capture the same level of information. Furthermore, they specify locations with the same level of precision. Both Cartesian coordinates and polar coordinates are capable of accurately describing any twodimensional relation. What differentiates Cartesian coordinates from polar coordinates is how they specify the relevant information — and, perhaps, what functional goals they serve.

After all, it is easy to imagine how distinct, redundant formats may serve flexible spatial behavior. If one 
is tasked with getting directly from point A to point B, a graph-like, polar-esque format may be most apt. If instead one is tasked with representing the full layout of a city, a Euclidean, Cartesian format may be most apt. Finally, if one is tasked with communicating directions to another person, a propositional format may prove useful; it is much easier to say "it is down that street just past the coffee shop" than it is to convey any exact distance or direction (in the same way it is easier to use algebraic notation to describe a game of chess). The advantage of redundant formats is that we need not constantly translate information from one 'code' into another; on this view, it is possible to automatically store information in multiple formats which can be individually called upon depending on the needs of the current task.

Of course, the jury is still out on whether we do in fact represent space in multiple formats simultaneously. While the available evidence suggests this may be the case, there is no definitive indication of this, to date. Future work ought to keep in mind the possibility that space may be represented in multiple formats (rather than tirelessly trying to reduce behavior to a single one).

The spirit of this argument extends far beyond spatial representation. In all other domains, one of the goals of cognitive science is to characterize the primitive representations that bridge the gap between brain and behavior. Whether we study memory, or social relations, or concepts, or number — or anything else! - we share a fundamental goal to understand the format of information in the human mind. And in all of these cases, (1) these representations may ultimately be formatted in a spatial way (see Peer et al., 2020), and (2) these representations may be formatted in multiple ways at once. If this is true, any search for a single, ultimate format may be misguided.

\subsection{Other Spatial Formats}

Location representations are not the only kind of spatial representation for which we may think about 'format'. The notion that the mind represents information in discernible formats - and may utilize multiple, 'redundant' formats - applies as well to spatial properties like shape, size, and orientation.

For example, work in vision science has tried to identify the format of shape representations that support object recognition (e.g., Biederman, 1987; Biederman \& Bar, 1999, Kanizsa, 1976; Leyton, 1989). Inspired by work in computer vision (e.g., Liu \& Gieger, 1999; Shokoufandeh et al., 2005; see also Blum, 1973), it has been suggested that shapes in the human mind are represented as 'shape skeletons' via the medial axis (which describes the set of all points within an object having two or more closest points along the perimeter of that object; see Psotka, 1978). Given the convergence of behavioral and neuroscientific evidence (see also Ayzenberg \& Lourenco, 2019; Lowet et al., 2018), shape skeletons - and the medial axis in particular provide one of the most robust examples of format in human cognition. 
Shape skeletons like medial axes are often compared to other models of shape representation, like principal axes (as in Ayzenberg et al., 2019; Firestone \& Scholl, 2014); indeed, medial axes often out-perform other possible formats. However, here we might fruitfully apply this notion that representations need not be reduced to a single format. There is, after all, work supporting the role of principal axes (Marr \& Nishihara, 1978; Sturz, Boyer, Magnotti, \& Bodily, 2017). As with location representations, it may be that the mind utilizes distinct formats in different ways depending on the task. For instance, it may be that the medial axis is used for object recognition, but that the principal axis is used to evaluate rough size (e.g., whether a certain piece of furniture will fit in a certain space).

These same insights can be applied to the study of other spatial properties, like area (see Corbett \& Oriet, 2011; Marchant et al., 2013; Raidvee et al., 2020; Solomon et al., 2011; Yousif, Aslin, \& Keil, 2020; Yousif \& Keil, 2019; Yousif \& Keil, 2021a), volume (Bennette, Keil, \& Yousif, 2021; Ekman \& Junge, 1961; Teghtsoonian, 1965), and orientation (see Appelle, 1972; Lee et al., 2003; Girschick et al., 2011; Henderson \& Serences, 2021; Sadalla \& Montello, 1989; Yousif, Chen, \& Scholl, 2020). In all of these cases, it may be useful to consider the possibility that any piece of information may be formatted in multiple ways simultaneously.

\subsection{Conclusion}

The 'format' of many mental representations is surprisingly accessible: whether studying ants in the Tunisian desert or human visual localization in the lab, 'format' often reveals itself in ordinary behavior. Even tiny, almost-imperceptible errors provide valuable insight into how the mind works. While much is known about the nature of spatial representations, many foundational questions are yet to be answered. Here, I have considered one such question: Must all cognitive representations be reduced to a single format, or is it possible that the mind represents some information in multiple formats simultaneously? For various spatial properties, there are indeed reasons to believe that space may by default be represented in multiple formats - and that this seemingly redundant form of representation may be what allows for flexible spatial behavior in the first place. There are also reasons to believe that some common formats underlie representations across modalities. These findings collectively provide a hint at how adaptable, complex spatial behavior may arise from simple, low-dimensional representations.

Moving forward, it may benefit the field not to think of cognitive representations as being reducible into one, most-primitive format, but instead to think of the myriad of ways the mind may format a given piece of information at once (and the goals each format would serve). And insofar as spatial representation may serve as the foundation for other higher-level cognitive representations (number, social relationships, etc.), we may use these insights to better understand not just spatial representation but all mental representation. 


\section{Acknowledgments}

This paper was made possible in part by an NSF Graduate Research Fellowship. For helpful feedback and discussion, I thank Dick Aslin, Lauren Aulet, Vlad Ayzenberg, Danny Dilks, Sarah Joo, Frank Keil, Josh Knobe, Stella Lourenco, Sam McDougle, Darko Odic, Brynn Sherman, and Flora Zhang. I would especially like to thank Frank Keil for his tireless support the past five years. This paper was made possible because Frank agreed to advise a student with research interests very different from his own. Frank pushed me and challenged me and inspired me, all while being unreasonably patient and incredibly kind on my (many) bad days. All people should aspire to be as open-minded and as generous and as selfless as Frank Keil. 


\section{References}

Appelle, S. (1972). Perception and discrimination as a function of stimulus orientation: The "oblique effect" in man and animals. Psychological Bulletin, 78, 266-278.

Aulet, L. S., Yousif, S. R., \& Lourenco, S. F. (2021). Spatial-numerical associations from a novel paradigm support the mental number line account. Quarterly Journal of Experimental Psychology, 74, 1829-1840.

Ayzenberg, V., Chen, Y., Yousif, S. R., \& Lourenco, S. F. (2019). Skeletal representations of shape in human vision: Evidence for a pruned medial axis model. Journal of Vision, 19, 1-21.

Ayzenberg, V., \& Lourenco, S. F. (2019). Skeletal descriptions of shape provide unique perceptual information for object recognition. Scientific Reports, 9, 1-13.

Baud-Bovy, G., \& Viviani, P. (1998). Pointing to kinesthetic targets in space. Journal of Neuroscience, 18, $1528-1545$.

Bennette, E., Keil, F. C., \& Yousif, S. R. (2021). A Ubiquitous Illusion of Volume: Are Impressions of 3D Volume Captured by an "Additive Heuristic"? Perception, 50, 462-469.

Bicanski, A., \& Burgess, N. (2019). A computational model of visual recognition memory via grid cells. Current Biology, 29, 979-990.

Biederman, I. (1987). Recognition-by-components: a theory of human image understanding. Psychological Review, 94, 115-147.

Biederman, I., \& Bar, M. (1999). One-shot viewpoint invariance in matching novel objects. Vision Research, $39,2885-2899$.

Brooks, R. A. (1991). Intelligence without representation. Artificial Intelligence, 47, 139-159.

Butler, W. N., Hardcastle, K., \& Giocomo, L. M. (2019). Remembered reward locations restructure entorhinal spatial maps. Science, 363(6434), 1447-1452.

Chang, L., \& Tsao, D. Y. (2017). The code for facial identity in the primate brain. Cell, 169, 1013-1028.

Chen, X., He, Q., Kelly, J. W., Fiete, I. R., \& McNamara, T. P. (2015). Bias in human path integration is predicted by properties of grid cells. Current Biology, 25, 1771-1776.

Cheng, K. (1986). A purely geometric module in the rat's spatial representation. Cognition, 23, 149-178.

Cheng, K., \& Newcombe, N. S. (2005). Is there a geometric module for spatial orientation? Squaring theory and evidence. Psychonomic Bulletin \& Review, 12, 1-23.

Chown, E., Kaplan, S., \& Kortenkamp, D. (1995). Prototypes, location, and associative networks (PLAN): Towards a unified theory of cognitive mapping. Cognitive Science, 19, 1-51.

Corbett, J. E., \& Oriet, C. (2011). The whole is indeed more than the sum of its parts: Perceptual averaging in the absence of individual item representation. Acta Psychologica, 138, 289-301. 
Dehaene, S., Bossini, S., \& Giraux, P. (1993). The mental representation of parity and number magnitude. Journal of Experimental Psychology: General, 122, 371-396.

Ekman, G., \& Junge, K. (1961). Psychophysical relations in visual perception of length, area and volume. Scandinavian Journal of Psychology, 2, 1-10.

Firestone, C., \& Scholl, B. J. (2014). "Please tap the shape, anywhere you like" shape skeletons in human vision revealed by an exceedingly simple measure. Psychological Science, 25, 377-386.

Flanders, M., Tillery, S. I. H., \& Soechting, J. F. (1992). Early stages in a sensorimotor transformation. Behavioral and Brain Sciences, 15, 309-320.

Gallistel, C. R. (1990). The organization of learning. Cambridge, MA: MIT press.

Girshick, A. R., Landy, M. S., \& Simoncelli, E. P. (2011). Cardinal rules: visual orientation perception reflects knowledge of environmental statistics. Nature Neuroscience, 14, 926-932.

Gordon, J., Ghilardi, M. F., Cooper, S. E., \& Ghez, C. (1994). Accuracy of planar reaching movements. Experimental Brain Research, 99, 112-130.

Hafting, T., Fyhn, M., Molden, S., Moser, M. B., \& Moser, E. I. (2005). Microstructure of a spatial map in the entorhinal cortex. Nature, 436, 801-806.

Hegarty, M., Montello, D. R., Richardson, A. E., Ishikawa, T., \& Lovelace, K. (2006). Spatial abilities at different scales: Individual differences in aptitude-test performance and spatial-layout learning. Intelligence, 34, 151-176.

Henderson, M. M., \& Serences, J. (2021). Biased orientation representations can be explained by experience with non-uniform training set statistics. bioRxiv, 2020-07.

Hermer, L., \& Spelke, E. S. (1994). A geometric process for spatial reorientation in young children. Nature, $370,57-59$.

Holden, M. P., Newcombe, N. S., \& Shipley, T. F. (2013). Location memory in the real world: Category adjustment effects in 3-dimensional space. Cognition, 128, 45-55.

Holyoak, K. J., \& Mah, W. A. (1982). Cognitive reference points in judgments of symbolic magnitude. Cognitive Psychology, 14, 328-352.

Hudson, T. E., \& Landy, M. S. (2016). Sinusoidal error perturbation reveals multiple coordinate systems for sensorymotor adaptation. Vision Research, 119, 82-98.

Huttenlocher, J., Hedges, L. V., \& Duncan, S. (1991). Categories and particulars: prototype effects in estimating spatial location. Psychological Review, 98, 352.

Huttenlocher, J., Newcombe, N., \& Sandberg, E. H. (1994). The coding of spatial location in young children. Cognitive Psychology, 27, 115-147. 
Jiang, Y., Olson, I. R., \& Chun, M. M. (2000). Organization of visual short-term memory. Journal of Experimental Psychology: Learning, Memory, and Cognition, 26, 683-702.

Julian, J. B., Keinath, A. T., Frazzetta, G., \& Epstein, R. A. (2018). Human entorhinal cortex represents visual space using a boundary-anchored grid. Nature Neuroscience, 21, 191-194.

Kanizsa, G. (1976). Subjective contours. Scientific American, 234, 48-53.

Kosslyn, S. M. (1996). Image and brain: The resolution of the imagery debate. MIT press.

Kosslyn, S. M., Koenig, O., Barrett, A., Cave, C. B., Tang, J., \& Gabrieli, J. D. (1989). Evidence for two types of spatial representations: Hemispheric specialization for categorical and coordinate relations. Journal of Experimental Psychology: Human Perception and Performance, 15, 723-735.

Kosslyn, S. M., Chabris, C. F., Marsolek, C. J., \& Koenig, O. (1992). Categorical versus coordinate spatial relations: computational analyses and computer simulations. Journal of Experimental Psychology: Human Perception and Performance, 18, 562-577.

Kosslyn, S. M., Thompson, W. L., Klm, I. J., \& Alpert, N. M. (1995). Topographical representations of mental images in primary visual cortex. Nature, 378, 496-498.

Kuipers, B. (1978). Modeling spatial knowledge. Cognitive Science, 2, 129-153.

Kuipers, B. (1982). The" map in the head" metaphor. Environment and Behavior, 14, 202-220.

Learmonth, A. E., Newcombe, N. S., Sheridan, N., \& Jones, M. (2008). Why size counts: Children's spatial reorientation in large and small enclosures. Developmental Science, 11, 414-426.

Lee, S. A., Sovrano, V. A., \& Spelke, E. S. (2012). Navigation as a source of geometric knowledge: Young children's use of length, angle, distance, and direction in a reorientation task. Cognition, 123, 144-161.

Leyton, M. (1989). Inferring causal history froms shape. Cognitive Science, 13, 357-387.

Liu, T. L., \& Geiger, D. (1999, September). Approximate tree matching and shape similarity. In Proceedings of the Seventh IEEE International Conference on Computer Vision (Vol. 1, pp. 456-462). IEEE.

Lowet, A. S., Firestone, C., \& Scholl, B. J. (2018). Seeing structure: Shape skeletons modulate perceived similarity. Attention, Perception, \& Psychophysics, 80, 1278-1289.

MacDonald, C. J., Lepage, K. Q., Eden, U. T., \& Eichenbaum, H. (2011). Hippocampal "time cells" bridge the gap in memory for discontiguous events. Neuron, 71, 737-749.

Marchant, A. P., Simons, D. J., \& de Fockert, J. W. (2013). Ensemble representations: Effects of set size and item heterogeneity on average size perception. Acta Psychologica, 142, 245-250.

Maley, C. J. (2021, October 5). The physicality of representation. https://doi.org/10.31234/osf.io/ame6u

Markman, A. B., \& Dietrich, E. (2000). In defense of representation. Cognitive Psychology, 40, 138-171.

Marr, D. (1982). Vision. A Computational Investigation into the Human Representation and Processing of Visual Information, W. H. Freeman, San Francisco. 
Marr, D., \& Nishihara, H. K. (1978). Representation and recognition of the spatial organization of threedimensional shapes. Proceedings of the Royal Society of London. Series B. Biological Sciences, 200, 269-294.

McCloskey, M., \& Palmer, E. (1996). Visual representation of object location: Insights from localization impairments. Current Directions in Psychological Science, 5, 25-28.

McCloskey, M., Rapp, B., Yantis, S., Rubin, G., Bacon, W. F., Dagnelie, G., Gordon, B., Aliminosa, D., Boatman, D. F., Badecker, W., Johnson, D. N., Tusa, R. J., \& Palmer, E. (1995). A developmental deficit in localizing objects from vision. Psychological Science, 6, 112-117.

McNamara, T. P., Hardy, J. K., \& Hirtle, S. C. (1989). Subjective hierarchies in spatial memory. Journal of Experimental Psychology: Learning, Memory, and Cognition, 15, 211.

Meilinger, T. (2008, September). The network of reference frames theory: A synthesis of graphs and cognitive maps. In International conference on spatial cognition (pp. 344-360). Springer, Berlin, Heidelberg.

Messier, J., \& Kalaska, J. F. (1999). Comparison of variability of initial kinematics and endpoints of reaching movements. Experimental Brain Research, 125, 139-152.

Moser, E. I., Kropff, E., \& Moser, M. B. (2008). Place cells, grid cells, and the brain's spatial representation system. Annual Reviews Neuroscience. 31, 69-89.

Müller, M., \& Wehner, R. (1988). Path integration in desert ants, Cataglyphis fortis. Proceedings of the National Academy of Sciences, 85, 5287-5290.

O'Keefe, J., \& Dostrovsky, J. (1971). The hippocampus as a spatial map: Preliminary evidence from unit activity in the freely-moving rat. Brain Research, 34, 171-175.

O'Keefe, J., \& Nadel, L. (1978). The hippocampus as a cognitive map. Oxford: Clarendon Press.

Palmer, S. E., Schloss, K. B., \& Sammartino, J. (2013). Visual aesthetics and human preference. Annual Review of Psychology, 64, 77-107.

Paivio, A. (1991). Dual coding theory: Retrospect and current status. Canadian Journal of Psychology, 45, $255-287$.

Park, S. A., Miller, D. S., Nili, H., Ranganath, C., \& Boorman, E. D. (2020). Map making: constructing, combining, and inferring on abstract cognitive maps. Neuron, 10\%, 1226-1238.

Parkinson, C., \& Wheatley, T. (2013). Old cortex, new contexts: Re-purposing spatial perception for social cognition. Frontiers in Human Neuroscience, 7, 1-7.

Peer, M., Brunec, I. K., Newcombe, N. S., \& Epstein, R. A. (2020). Structuring Knowledge with Cognitive Maps and Cognitive Graphs. Trends in Cognitive Sciences. 25. 37-54.

Poucet, B. (1993). Spatial cognitive maps in animals: new hypotheses on their structure and neural mechanisms. Psychological Review, 100, 163. 
Pylyshyn, Z. W. (1973). What the mind's eye tells the mind's brain: A critique of mental imagery. Psychological Bulletin, 80, 1-24.

Pylyshyn, Z. W. (2002). Mental imagery: In search of a theory. Behavioral and Brain Sciences, 25, 157-238.

Psotka, J. (1978). Perceptual processes that may create stick figures and balance. Journal of Experimental Psychology: Human Perception and Performance, 4, 101-111.

Raidvee, A., Toom, M., Averin, K., \& Allik, J. (2020). Perception of means, sums, and areas. Attention, Perception, 83 Psychophysics, 82, 865-876.

Robinson, D. A. (1972). Eye movements evoked by collicular stimulation in the alert monkey. Vision Research, 12, 1795-1808.

Sadalla, E. K., Burroughs, W. J., \& Staplin, L. J. (1980). Reference points in spatial cognition. Journal of Experimental Psychology: Human Learning and Memory, 6, 516.

Sadalla, E. K., \& Montello, D. R. (1989). Remembering changes in direction. Environment and Behavior, 21, 346-363.

Saffran, J. R., Aslin, R. N., \& Newport, E. L. (1996). Statistical learning by 8-month-old infants. Science, 274, 1926-1928.

Shelton, A. L., \& McNamara, T. P. (1997). Multiple views of spatial memory. Psychonomic Bulletin $\&$ Review, 4, 102-106.

Shokoufandeh, A., Macrini, D., Dickinson, S., Siddiqi, K., \& Zucker, S. W. (2005). Indexing hierarchical structures using graph spectra. IEEE Transactions on Pattern Analysis and Machine Intelligence, 27, $1125-1140$.

Solomon, J. A., Morgan, M., \& Chubb, C. (2011). Efficiencies for the statistics of size discrimination. Journal of Vision, 11, 1-11.

Stevens, S. S., \& Guirao, M. (1963). Subjective scaling of length and area and the matching of length to loudness and brightness. Journal of Experimental Psychology, 66, 177-186.

Sturz, B. R., Boyer, T. W., Magnotti, J. F., \& Bodily, K. D. (2017). Do Eye Movements During Shape Discrimination Reveal an Underlying Geometric Structure? Animal Behavior and Cognition, 4, 267-285.

Taube, J. S. (1998). Head direction cells and the neurophysiological basis for a sense of direction. Progress in Neurobiology, 55, 225-256.

Taylor, H. A., \& Tversky, B. (1992). Descriptions and depictions of environments. Memory \& Cognition, 20, 483-496.

Teghtsoonian, M. (1965). The judgment of size. The American Journal of Psychology, 78, 392-402.

Thelen, E., \& Smith, L. B. (1996). A dynamic systems approach to the development of cognition and action. MIT press. 
Tolman, E. C. (1948). Cognitive maps in rats and men. Psychological Review, 55, 189-208.

Theves, S., Fernandez, G., \& Doeller, C. F. (2019). The hippocampus encodes distances in multidimensional feature space. Current Biology, 29, 1226-1231.

Traxler, M., \& Gernsbacher, M. A. (Eds.). (2011). Handbook of psycholinguistics. Elsevier.

Tversky, B. (1993, September). Cognitive maps, cognitive collages, and spatial mental models. In European Conference on Spatial Information Theory (pp. 14-24). Springer, Berlin, Heidelberg.

Twyman, A. D., \& Newcombe, N. S. (2010). Five reasons to doubt the existence of a geometric module. Cognitive Science, 34, 1315-1356.

Waller, D., Montello, D. R., Richardson, A. E., \& Hegarty, M. (2002). Orientation specificity and spatial updating of memories for layouts. Journal of Experimental Psychology: Learning, Memory, and Cognition, 28, 1051 .

Warren, W. H., Rothman, D. B., Schnapp, B. H., \& Ericson, J. D. (2017). Wormholes in virtual space: From cognitive maps to cognitive graphs. Cognition, 166, 152-163.

Wittlinger, M., Wehner, R., \& Wolf, H. (2006). The ant odometer: stepping on stilts and stumps. Science, 312, 1965-1967.

van Dijck, J.-P., Abrahamse, E. L., Acar, F., Ketels, B., \& Fias, W. (2014). A working memory account of the interaction between numbers and spatial attention. Quarterly Journal of Experimental Psychology, $67,1500-1513$.

van Dijck, J. P., \& Fias, W. (2011). A working memory account for spatial-numerical associations. Cognition, $119,114-119$.

Xing, J., \& Andersen, R. A. (2000). Models of the posterior parietal cortex which perform multimodal integration and represent space in several coordinate frames. Journal of Cognitive Neuroscience, 12, 601-614.

Yang, F., \& Flombaum, J. (2018). Polar coordinates as the format of spatial representation in visual perception. Journal of Vision, 18, 21. https://doi.org/10.1167/18.10.21

Yousif, S., Aslin, R., \& Keil, F. (2020). Judgments of spatial extent are fundamentally illusory: 'Additivearea' provides the best explanation. Cognition. 205. 104439.

Yousif, S. R., \& Keil, F. C. (2019). The additive-area heuristic: An efficient but illusory means of visual area approximation. Psychological Science, 30, 495-503.

Yousif, S. R., \& Keil, F. C. (2021a). How we see area and why it matters. Trends in Cognitive Sciences. 25, 554-557.

Yousif, S. R., \& Keil, F. C. (2021b). The shape of space: Evidence for spontaneous but flexible use of polar coordinates in visuospatial representations. Psychological Science, 32, 573-586. 
Yousif, S. R., \& Lourenco, S. F. (2017). Are all geometric cues created equal? Children's use of distance and length for reorientation. Cognitive Development, 43, 159-169.

Yousif, S. R., Rosenberg, M. D., \& Keil, F. C. (2021). Using space to remember: Short-term spatial structure spontaneously improves working memory. Cognition, 214, 104748. 